\title{
Note About Hamiltonian Dynamics of String in Non-Relativistic D3-Brane Background
}

\author{
by J. Klusoň \\ Department of Theoretical Physics and Astrophysics \\ Faculty of Science, Masaryk University \\ Kotlářská 2, 611 37, Brno \\ Czech Republic \\ E-mail: klu@physics.muni.cz
}

\begin{abstract}
This paper is devoted to the study of the Hamiltonian dynamics of string in non-relativistic D3-brane background. We discuss different gauge fixing functions and construct corresponding gauge fixed Hamiltonians.
\end{abstract}

Keywords: Bosonic String, Non-relativistic Background. 


\section{Contents}

1. Introduction and Summary 1

2. Hamiltonian Formalism for String in Non-Relativistic D3-brane Background

3. Static Gauge 6

3.1 Static Gauge $t=\tau, r=\sigma$

3.2 Static Gauge $t=\tau, \xi=\sigma$

4. Uniform Gauge $t=\tau, p_{\xi}=$ const 10

5. Strings in Non-Extremal Non-Relativistic D3-brane Background 13

\section{Introduction and Summary}

The AdS/CFT correspondence relates conformal field theories in $d$-dimensional flat spacetimes to gravitational theories (super strings) in asymptotically $A d S_{d+1}$ spacetimes [1, 2, 3]. Recently this correspondence has been generalized to the description of some nonrelativistic strongly coupled conformal systems ${ }^{1}$. Non-relativistic conformal symmetry contains the scaling transformation

$$
x^{i}=\lambda x^{i}, \quad t^{\prime}=\lambda^{z} t,
$$

where $z$ is a dynamical exponent. In case of $z=2$ this symmetry is enhanced to Schorödinger symmetry [6, 0, 8, 9, 10, 11, 12, 13, 14, 15]. It is remarkable that it is possible to find the gravity dual of these non-relativistic field theories [16, 17] ${ }^{2}$. The asymptotic metric in this case reads

$$
d s^{2}=\frac{R^{2}}{r^{2}}\left(-\frac{d t^{2}}{r^{2(z-1)}}+2 d t d \xi+\left(d x^{i}\right)^{2}\right)+\frac{R^{2}}{r^{2}} d r^{2}+d s_{M}^{2},
$$

where $R$ is characteristic radius of space-time, $\xi \sim \xi+L$ is a compact light-like coordinate and where $d s_{M}^{2}$ is the metric of an appropriate compact manifold which allows (1.2) to be a solution to the supergravity equations of motion. Since $\xi$ is compact the associated quantum number is interpreted as the particle number.

As we said above $z$ that appears in the scaling relation (1.1)is a critical exponent. The usual AdS case corresponds to $z=1$. On the other hand non-relativistic Dp-brane

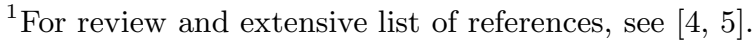

${ }^{2}$ For another solutions that should be dual to non-relativistic field theories, see 18, 19, 20, 21, 22, 23, $24,25,26,27,28,29,30,31,32,33,34,35,36,37,38,39,39,41,42,43,44,45,46,47,48,49,50,51,52$.
} 
backgrounds are characterized by dynamical exponents $z=2$ [21, 26]. The backgrounds with more general values of $z$ were also found however it is still an open question of their consistency, for nice discussion, see [32]. For example, while Schrödinger space-time is geodetically complete at $r \rightarrow 0$ for all $z \geq 1$, for $z>1$ the detailed behavior of geodesics near $r=0$ differs somewhat from the case $z=1$ since ( $r=0$ is harder to reach), and this may well have implications for holography and, in particular, for an appropriate notion of boundary in this context.

Since the holographic relation between non-relativistic theory on boundary and the string theory in the bulk is not complete developed it is certainly useful to implement ideas from standard $A d S / C F T$ correspondence to this case in order to gain insight into the subject ${ }^{3}$. For example, it is well known that the dynamics of the string in uniform gauge where momentum along compact direction is uniformly distributed on the string is useful tool for the study of the relations between observables in CFT on the boundary and the string states in the bulk of $A d S_{5}$ [54], for recent review, see [55]. Since the extremal non-relativistic background is characterized by an existence of compact light-like direction $\xi$ and off-diagonal metric component $g_{t \xi}$ together with an absence of $g_{\xi \xi}$ we mean that it makes sense to study the Hamiltonian dynamics of the string in this background where different gauge fixing conditions are imposed. We will see that in the standard gauge when the world-sheet time coincides with target space time the Hamiltonian dynamics is well defined on condition of non-zero momentum along $\xi$ direction. More precisely, we consider two gauge fixing: The first one when we demand that the world-sheet coordinates coincide with target space ones and the second one when we demand that the worldsheet time coincides with the background time and when the momentum $P_{\xi}$ is uniformly distributed along the world-sheet of the string. In both these cases we find that gauge fixed Hamiltonians have manifestly non-relativistic form. We also argue that in the gauge when the string wraps $\xi$ direction there is no pure time dependent solutions of the equations of motion. On the other hand in case of uniform gauge there is a possibility of pure time dependent dynamics and we find that the string with finite energy cannot reach the boundary of space time at $r=0$ which is in agreement with previous analysis of radial geodesics in Schrödinger spacetimes [32]. Finally we construct the string in uniform gauge in the non-extremal non-relativistic background and we find the Hamiltonian density has "square-root" form as in case of relativistic background.

This paper can be extended in many directions. For example, it will be interesting to study the static solutions corresponding to Wilson lines. We can also search for more general configurations of the string as for example folded or pulsating strings with analogy with solutions found in the context of AdS/CFT correspondence (For review, see [56, 57]).

This paper is organized as follows. In section (2) we review the Hamiltonian dynamics of the bosonic string in general background. In section (3) we consider the static gauge for the bosonic string in non-relativistic background and we find the gauge fixed form of the Hamiltonian density. In section (4) we study the uniform gauge and we also study the dynamics of the radial mode. Finally in section (5) we find the uniform gauge fixed form

\footnotetext{
${ }^{3}$ Very nice discussion of the non-relativistic holography can be found in 53
} 
of the Hamiltonian for the string in non-extremal non-relativistic D3-brane background.

\section{Hamiltonian Formalism for String in Non-Relativistic D3-brane Back- ground}

In this section we review the construction of the Hamiltonian for the bosonic string in

general background. Recall that the action for the bosonic string in general background takes the form

$$
S=-\frac{1}{4 \pi \alpha^{\prime}} \int d^{2} \sigma \sqrt{-\gamma}\left(\gamma^{\alpha \beta} g_{M N} \partial_{\alpha} x^{M} \partial_{\beta} x^{N}-\epsilon^{\alpha \beta} b_{M N} \partial_{\alpha} x^{M} \partial_{\beta} x^{N}\right),
$$

where $\gamma_{\alpha \beta}$ is a two-dimensional world-sheet metric, $\sigma^{\alpha}, \alpha, \beta=0,1, \sigma^{0}=\tau, \sigma^{1}=\sigma$ are world-sheet coordinates and $\epsilon^{\alpha \beta}=\frac{\varepsilon^{\alpha \beta}}{\sqrt{-\gamma}}, \varepsilon^{\tau \sigma}=-\varepsilon^{\sigma \tau}=1$. Further, $x^{M}, M, N=$ $0, \ldots, 9$ are modes that parameterize the embedding of the string into target space-time with background metric $g_{M N}$ and NS two-form $b_{M N}$.

Our goal is to develop the Hamiltonian formalism from the action (2.1). It is convenient to use following parametrization of the world-sheet metric

$$
\gamma_{\alpha \beta}=\left(\begin{array}{cc}
-N_{T}^{2}+\omega N_{S}^{2} & \omega N_{S} \\
\omega N_{S} & \omega
\end{array}\right)
$$

so that

$$
\operatorname{det} \gamma=-N_{T}^{2} \omega, \quad \gamma^{\alpha \beta}=\left(\begin{array}{cc}
-\frac{1}{N_{T}^{2}} & \frac{N_{S}}{N_{T}^{2}} \\
\frac{N_{S}}{N_{T}^{2}} & \frac{1}{\omega}-\frac{N_{S}^{2}}{N_{T}^{2}}
\end{array}\right) .
$$

With this form of the world-sheet metric the action (2.1) takes the form

$$
\begin{aligned}
S & =\frac{1}{4 \pi \alpha^{\prime}} \int d^{2} \sigma N_{T} \sqrt{\omega}\left(g_{M N} \nabla_{\tau} x^{M} \nabla_{\tau} x^{N}-\frac{1}{\omega} g_{M N} \partial_{\sigma} x^{M} \partial_{\sigma} x^{N}+\right. \\
& \left.+b_{M N} \partial_{\tau} x^{M} \partial_{\sigma} x^{N}-b_{M N} \partial_{\sigma} x^{M} \partial_{\tau} x^{N}\right),
\end{aligned}
$$

where

$$
\nabla_{\tau} x^{M}=\frac{1}{N_{T}}\left(\partial_{\tau} x^{M}-N_{S} \partial_{\sigma} x^{M}\right) .
$$

Now we introduce the momenta $\pi_{T}, \pi_{S}, \pi_{\omega}$ conjugate to $N_{T}, N_{S}$ and $\omega$ with canonical Poisson brackets

$$
\left\{N_{T}(\sigma), \pi_{T}\left(\sigma^{\prime}\right)\right\}=\delta\left(\sigma-\sigma^{\prime}\right), \quad\left\{N_{S}(\sigma), \pi_{S}\left(\sigma^{\prime}\right)\right\}=\delta\left(\sigma-\sigma^{\prime}\right), \quad\left\{\omega(\sigma), \pi_{\omega}\left(\sigma^{\prime}\right)\right\}=\delta\left(\sigma-\sigma^{\prime}\right) .
$$

Then due to the fact that the action (2.4) does not contain the time derivative of metric we find that these momenta are primary constraints of the theory

$$
\pi_{T}=\frac{\delta S}{\delta \partial_{\tau} N_{T}} \approx 0, \quad \pi_{S}=\frac{\delta S}{\delta \partial_{\tau} N_{S}} \approx 0, \quad \pi_{\omega}=\frac{\delta S}{\delta \partial_{\tau} \omega} \approx 0
$$


As the next step we introduce the momenta $p_{M}$ conjugate to $x^{M}$ so that $\left\{x^{M}(\sigma), p_{N}\left(\sigma^{\prime}\right)\right\}=$ $\delta_{N}^{M} \delta\left(\sigma-\sigma^{\prime}\right)$. From (2.4) we get

$$
p_{M}=\frac{\delta S}{\delta \partial_{\tau} x^{M}}=\frac{1}{2 \pi \alpha^{\prime}}\left(\sqrt{\omega} g_{M N} \nabla_{\tau} x^{N}+b_{M N} \partial_{\sigma} x^{N}\right) .
$$

Then it is easy to find the Hamiltonian density in the form

$$
\mathcal{H}=\partial_{\tau} x^{N} p_{M}-\mathcal{L}=\frac{N_{T}}{\sqrt{\omega}} \mathcal{H}_{T}+N_{S} \mathcal{H}_{S}
$$

where

$$
\begin{aligned}
& \mathcal{H}_{T}=\frac{1}{4 \pi \alpha^{\prime}}\left(\left(2 \pi \alpha^{\prime} p_{M}-b_{N K} \partial_{\sigma} x^{K}\right) g^{M N}\left(2 \pi \alpha^{\prime} p_{N}-b_{N L} \partial_{\sigma} x^{L}\right)+\partial_{\sigma} x^{M} g_{M N} \partial_{\sigma} x^{N}\right), \\
& \mathcal{H}_{S}=\partial_{\sigma} x^{N} p_{N} .
\end{aligned}
$$

Using the Hamiltonian (2.9) we easily determine the time evolution of the primary constraints as

$$
\begin{aligned}
& \partial_{\tau} \pi_{T}=\left\{\pi_{T}, H\right\}=-\frac{1}{\sqrt{\omega}} \mathcal{H}_{T}, \\
& \partial_{\tau} \pi_{\omega}=\left\{\pi_{\omega}, H\right\}=\frac{N_{T}}{\omega^{3 / 2}} \mathcal{H}_{T}, \\
& \partial_{\tau} \pi_{S}=\left\{\pi_{S}, H\right\}=-\mathcal{H}_{S} .
\end{aligned}
$$

Since the constraints (2.7) have to be preserved during the time evolution of the system the equations above imply an existence of the secondary constraints

$$
\mathcal{H}_{T} \approx 0, \quad \mathcal{H}_{S} \approx 0
$$

Finally it can be shown that the constraints $\mathcal{H}_{T}$ and $\mathcal{H}_{S}$ form the closed set of constraints and the consistency of their time evolution does not impose additional ones. In summary, we have collections of the first class constraints $\left(\pi_{T} \approx 0, \pi_{S} \approx 0, \pi_{\omega} \approx 0, \mathcal{H}_{T} \approx 0, \mathcal{H}_{S} \approx 0\right)$ and consequently the total Hamiltonian density is equal to 4

$$
\mathcal{H}=\left(\frac{N_{T}}{\sqrt{\omega}}+f_{T}\right) \mathcal{H}_{T}+\left(N_{S}+f_{S}\right) \mathcal{H}_{S}+u_{T} \pi_{T}+u_{S} \pi_{S}+u_{\omega} \pi_{\omega} \approx 0
$$

where $f_{T}, f_{S}, u_{T}, u_{S}, u_{\omega}$ are arbitrary functions that generally depend on world-sheet coordinates and on the phase-space variables. We will see in next sections that these functions are uniquely specified when we introduce the gauge fixing functions that impose additional constraints on the theory.

\footnotetext{
${ }^{4}$ For reviews of the dynamics of constrained systems, see 61, 62, 63.
} 
In what follows we concentrate on the dynamics of the bosonic string in non-relativistic D3-brane background [26 ${ }^{5}$

$$
\begin{aligned}
d s^{2} & =\frac{R^{2}}{r^{2}}\left(-\frac{d t^{2}}{r^{2}}+2 d t d \xi+\left(d x^{i}\right)^{2}\right)+\frac{R^{2}}{r^{2}}\left(d r^{2}+r^{2}\left((d \chi+\mathcal{A})^{2}+d s_{P^{2}}^{2}\right)\right) \\
B & =\frac{R^{2}}{r^{2}}(d \chi+\mathcal{A}) \wedge d t
\end{aligned}
$$

where

$$
\begin{aligned}
\mathcal{A} & =\frac{1}{2} \sin ^{2} \mu \sigma_{3}, \\
d s_{P^{2}}^{2} & =d \mu^{2}+\frac{1}{4} \sin ^{2} \mu\left(\sigma_{1}^{2}+\sigma_{2}^{2}+\cos ^{2} \mu \sigma_{3}^{2}\right),
\end{aligned}
$$

and where $\sigma_{i}$ are $S U(2)$ left-invariant forms that satisfy

$$
d \sigma_{i}=-\frac{1}{2} \epsilon_{i j k} \sigma_{j} \wedge \sigma_{k} .
$$

Explicitly they have the form

$$
\begin{aligned}
& \sigma_{1}=\cos \psi d \theta+\sin \theta \sin \psi d \phi, \\
& \sigma_{2}=-\sin \psi d \theta+\sin \theta \cos \psi d \phi, \\
& \sigma_{3}=d \psi+\cos \theta d \theta
\end{aligned}
$$

For simplicity we introduce the symbol $y^{a}, a=1,2,3,4$ defined as $y \equiv(\mu, \psi, \theta, \phi)$ and write the line element of squashed $S^{5}$ as

$$
d s_{S^{5}}^{2}=d \chi^{2}+g_{a \chi} d y^{a} d \chi+g_{\chi a} d \chi d y^{a}+g_{a b} d y^{a} d y^{b}
$$

where the explicit form of metric components can be easily determined from (2.14) and (2.15). In the same way we write

$$
b_{\chi t}=-b_{t \chi}=\frac{1}{2} \frac{R^{2}}{r^{2}}, \quad b_{a t}=-b_{t a}=\frac{1}{2} \frac{R^{2}}{r^{2}} \mathcal{A}_{a} .
$$

Now we are ready to proceed to the construction of the gauge-fixed form of the bosonic string action on non-relativistic background. Recall that the Hamiltonian (2.13) is a collection of the first class constraints and hence vanishes on constraint surface. The gauge fixing of the first class constraints consists in introduction of additional constraints in the theory that have non-trivial Poisson brackets with the initial first class constraints on the constraint surface. Then the collections of the original first class constraints together with

\footnotetext{
${ }^{5}$ Clearly this analysis can be generalized to more general form of the non-relativistic background.
} 
the gauge fixing functions form the set of the second class constraints. The characteristic property of the second class constraints is that they can be explicitly solved and hence some degrees of freedom can be eliminated. It is also clear that we should replace the original Poisson brackets with the Dirac ones. However it turns out that for the gauge fixed functions that we use in this paper the Poisson brackets coincide with the Dirac ones.

Of course all these comments are valid for any theory with the first class constraints. However the crucial property of the extremal non-relativistic background is an absence of the metric component $g_{\xi \xi}$. Then using the form of the metric 2.14 we find

$$
g^{t t}=0, \quad g^{\xi \xi}=-\frac{g_{t t}}{g_{t \xi}^{2}}, \quad g^{t \xi}=\frac{1}{g_{t \xi}} .
$$

The fact that $g^{t t}$ is zero will have an important consequence for the construction of the gauge fixed Hamiltonian.

\section{Static Gauge}

In this section we study the gauges when world-sheet coordinates coincide with some target space coordinates. We consider two situations:

\subsection{Static Gauge $t=\tau, r=\sigma$}

This gauge is defined by introducing following two gauge fixing functions

$$
G_{1}: t-\tau=0, \quad G_{2}: r-\sigma=0
$$

Let us now show that these functions fix all gauge freedom of the theory. We firstly determine the Poisson brackets between $G_{1}, G_{2}$ and $\mathcal{H}_{T}, \mathcal{H}_{S}$. For the background (2.14) these constraints take the form

$$
\begin{aligned}
\mathcal{H}_{S} & =p_{t} \partial_{\sigma} t+p_{r} \partial_{\sigma} r+\mathcal{H}_{S}^{r . s . g .} \\
\mathcal{H}_{T} & =\frac{1}{4 \pi \alpha^{\prime}}\left(2\left(2 \pi \alpha^{\prime}\right)^{2} p_{t} g^{t \xi} p_{\xi}+\left(2 \pi \alpha^{\prime}\right)^{2} p_{r} g^{r r} p_{r}+g_{r r} \partial_{\sigma} r \partial_{\sigma} r+\right. \\
& \left.+g_{t t} \partial_{\sigma} t \partial_{\sigma} t+2 g_{t \xi} \partial_{\sigma} t \partial_{\sigma} \xi\right)+\mathcal{H}_{T}^{r . s . g .}
\end{aligned}
$$

where

$$
\begin{aligned}
\mathcal{H}_{T}^{r . s . g .} & =\frac{1}{4 \pi \alpha^{\prime}}\left(\left(2 \pi \alpha^{\prime}\right)^{2} p_{\xi} g^{\xi \xi} p_{\xi}-2\left(2 \pi \alpha^{\prime}\right)^{2}\left(b_{t a} \partial_{\sigma} y^{a}+b_{t \chi} \partial_{\sigma} \chi\right) g^{t \xi} p_{\xi}+\right. \\
& +\left(2 \pi \alpha^{\prime}\right)^{2} p_{\chi} g^{\chi \chi} p_{\chi}+2\left(2 \pi \alpha^{\prime}\right)^{2} p_{\chi} g^{\chi a} p_{a}+\left(2 \pi \alpha^{\prime}\right)^{2} p_{a} g^{a b} p_{b}+ \\
& +\frac{1}{4 \pi \alpha^{\prime}}\left(g_{i j} \partial_{\sigma} x^{i} \partial_{\sigma} x^{j}+g_{\chi \chi}\left(\partial_{\sigma} \chi\right)^{2}+2 g_{a \chi} \partial_{\sigma} \chi \partial_{\sigma} y^{a}+g_{a b} \partial_{\sigma} y^{a} \partial_{\sigma} y^{b}\right), \\
\mathcal{H}_{S}^{r . s . g .} & =p_{\xi} \partial_{\sigma} \xi+p_{\chi} \partial_{\sigma} \chi+p_{a} \partial_{\sigma} y^{a}+p_{i} \partial_{\sigma} x^{i},
\end{aligned}
$$


and where for simplicity of notation we ignore from the beginning terms proportional to $b_{\xi t} \partial_{\sigma} t, b_{a t} \partial_{\sigma} t$ that vanish in all gauges studied in this paper. It is easy to determine the Poisson brackets between $G_{1}, G_{2}$ and $\mathcal{H}_{T}, \mathcal{H}_{S}$

$$
\begin{aligned}
& \left\{G_{1}(\sigma), \mathcal{H}_{T}\left(\sigma^{\prime}\right)\right\}=2 \pi \alpha^{\prime} g^{t \xi} p_{\xi}(\sigma) \delta\left(\sigma-\sigma^{\prime}\right), \\
& \left\{G_{1}(\sigma), \mathcal{H}_{S}\left(\sigma^{\prime}\right)\right\}=\partial_{\sigma} t(\sigma) \delta\left(\sigma-\sigma^{\prime}\right) \approx 0, \\
& \left\{G_{2}(\sigma), \mathcal{H}_{T}\left(\sigma^{\prime}\right)\right\}=2 \pi \alpha^{\prime} g^{r r} p_{r}(\sigma) \delta\left(\sigma-\sigma^{\prime}\right), \\
& \left\{G_{2}(\sigma), \mathcal{H}_{S}\left(\sigma^{\prime}\right)\right\}=\partial_{\sigma} r(\sigma) \delta\left(\sigma-\sigma^{\prime}\right) \approx \delta\left(\sigma-\sigma^{\prime}\right),
\end{aligned}
$$

where $\approx 0$ means that these results hold on the constraint surface $G_{1}=G_{2}=\mathcal{H}_{T}=\mathcal{H}_{S}=$ 0. Clearly the matrix of Poisson brackets (3.4) is non-singular and hence the collection of the original first class constraints with the gauge fixed functions forms the system of the second class constraints. It is also easy to see that the Dirac brackets of the canonical variables on the reduced space coincide with the Poisson brackets.

Clearly we also have to fix the gauge freedom that is related to the generators $\pi_{S}, \pi_{T}$ and $\pi_{\omega}$ and we do it by imposing the condition

$$
G_{\pi_{T}}: N_{T}-1=0, \quad G_{\pi_{S}}: N_{S}=0, \quad G_{\pi_{\omega}}: \omega-1=0 .
$$

It is easy to see that Poisson brackets of these gauge fixed functions with the constraints $\pi_{T}, \pi_{S}, \pi_{\omega}$ are non-zero and again the collections of gauge fixed functions given in (3.5) together with the primary constraints (2.7) form the second class constraints.

We can also see that the functions (3.1) fix the gauge from the following arguments. The consistency of the theory implies that the conditions (3.1) have to be preserved during the time evolution of the system. Since the time evolution of any phase space function is governed by the equation

$$
\frac{d \Phi}{d \tau}=\partial_{\tau} \Phi+\{\Phi, H\}
$$

we find

$$
\begin{aligned}
\frac{d G_{1}}{d \tau} & =-1+\left(\frac{N_{T}}{\sqrt{\omega}}+f_{T}\right) 2 \pi \alpha^{\prime} g^{t \xi} p_{\xi}+\left(N_{S}+f_{S}\right) \partial_{\sigma} t \approx \\
& \approx-1+\left(1+f_{T}\right) 2 \pi \alpha^{\prime} g^{t \xi} p_{\xi}=0
\end{aligned}
$$

and hence $f_{T}$ is equal to

$$
f_{T}=\frac{1-2 \pi \alpha^{\prime} g^{t \xi} p_{\xi}}{2 \pi \alpha^{\prime} g^{t \xi} p_{\xi}} .
$$

This result also implies that the gauge fixed theory is well defined on condition that $p_{\xi} \neq 0$. On the other hand the requirement of the preservation of $G_{2}$ under the time evolution implies

$$
\begin{aligned}
\frac{d G_{2}}{d \tau} & =\left(\frac{N_{T}}{\sqrt{\omega}}+f_{T}\right) 2 \pi \alpha^{\prime} g^{r r} p_{r}+\left(N_{S}+f_{S}\right) \partial_{\sigma} r \approx \\
& \approx\left(1+f_{T}\right) 2 \pi \alpha^{\prime} g^{r r} p_{r}+f_{S}=0
\end{aligned}
$$


and with the help of (3.8) we get $f_{S}=-\frac{g^{r r}}{g^{t \xi}} \frac{p_{r}}{p_{\xi}}=-\frac{p_{r}}{p_{\xi}}$. Finally the preservation of the gauge fixing functions (3.5) implies that

$$
u_{\pi_{T}}=0, \quad u_{\pi_{S}}=0, \quad u_{\pi_{\omega}}=0 .
$$

To conclude, the result of the gauge fixing procedure is that the Hamiltonian density is strongly zero and that the original constraints together with the gauge fixing functions consist a collection of the second class constraints. Then solving the constraint $\mathcal{H}_{S}=0$ we find

$$
p_{r}=-\mathcal{H}_{S}^{\text {r.s.g. }} .
$$

Further, the Hamiltonian density of the gauge fixed theory is equal to $\mathcal{H}^{\text {r.s.g. }}=-p_{t}$. This identification can be nicely seen from the fact that the action of gauge fixed theory is

$$
S=\int d^{2} \sigma\left(\partial_{\tau} x^{M} p_{M}-\mathcal{H}\right)=\int d^{2} \sigma\left(p_{a} \partial_{\tau} y^{a}+p_{i} \partial_{\tau} x^{i}+p_{\xi} \partial_{\tau} \xi+p_{\chi} \partial_{\tau} \chi-p_{t}\right)
$$

where we used the fact that the original Hamiltonian density $\mathcal{H}$ strongly vanishes. The Hamiltonian density $\mathcal{H}^{\text {r.s.g. }}$ can be derived by solving the constraint $\mathcal{H}_{T}=0$

$$
\begin{aligned}
\mathcal{H}^{r . s . g .} & =-p_{t}=\frac{1}{8 \pi^{2} \alpha^{\prime 2} g^{t \xi} p_{\xi}}\left(\left(2 \pi \alpha^{\prime}\right)^{2} p_{r} g^{r r} p_{r}+g_{r r}+\mathcal{H}_{T}^{r . s . g .}\right)= \\
& =\frac{1}{8 \pi^{2} \alpha^{\prime 2} g^{t \xi} p_{\xi}}\left(\left(2 \pi \alpha^{\prime}\right)^{2}\left(\mathcal{H}_{S}^{r . s . g .}\right)^{2} g^{r r}+g_{r r}+\mathcal{H}_{T}^{r . s . g .}\right),
\end{aligned}
$$

where in the second step we used (3.11).

Clearly the static gauge can be also imposed in the Lagrangian formalism of the bosonic string however we mean that it was instructive to perform this analysis in the Hamiltonian formalism in order to introduce main ideas and notations. Note also that this Hamiltonian is well defined on condition that $p_{\xi} \neq 0$. We also see that the static gauge can be implemented for either an open string or for an infinite long string since the variable $r$ is non-periodic. In fact, string in the gauge $t=\tau, r=\sigma$ can be used for the description of the the Wilson loops in non-relativistic theories as in case of AdS/CFT correspondence [58, 59, 60].

\subsection{Static Gauge $t=\tau, \xi=\sigma$}

As in previous section we fix the primary constraints (2.7) with functions (3.5). Then we fix the constraints $\mathcal{H}_{T} \approx 0, \mathcal{H}_{S} \approx 0$ by introducing two conditions

$$
G_{t}: t-\tau=0, \quad G_{\xi}: \xi-m \sigma=0,
$$

where the number $m$ has following interpretation. We known that $\xi$ is a compact variable with the identification $\xi \sim \xi+L$. As a consequence the gauge (3.14) is defined for closed string where $\sigma$ belongs to the finite interval $\sigma \in[-r, r]$ and all world-sheet modes are 
periodic on given interval. The number $r$ can be determined from the gauge fixing condition (3.14) since

$$
\xi(r)-\xi(-r)=\int_{-r}^{r} d \sigma \partial_{\sigma} \xi=\int_{-r}^{r} d \sigma m=2 r m=m L
$$

and we see that for $r=\frac{1}{2} L$ the number $m$ is the wrapping number that counts how many times the string wraps the compact direction $\xi$.

In order to simplify the final results we will write the constraints $\mathcal{H}_{S}, \mathcal{H}_{T}$ in the form

$$
\begin{aligned}
\mathcal{H}_{S} & =p_{t} \partial_{\sigma} t+p_{\xi} \partial_{\sigma} \xi+\mathcal{H}_{S}^{\xi . g . f .} \\
\mathcal{H}_{T} & =\frac{1}{4 \pi \alpha^{\prime}}\left(4 \pi \alpha^{\prime}\left(2 \pi \alpha^{\prime} p_{t}-b_{t I} \partial_{\sigma} \mathbf{x}^{I}\right) g^{t \xi} p_{\xi}+\left(2 \pi \alpha^{\prime}\right)^{2} p_{\xi} g^{\xi \xi} p_{\xi}+\right. \\
& \left.+g_{t t} \partial_{\sigma} t \partial_{\sigma} t+2 g_{t \xi} \partial_{\sigma} t \partial_{\sigma} \xi\right)+\mathcal{H}_{T}^{\xi . g . f .}
\end{aligned}
$$

where

$$
\mathcal{H}_{S}^{\xi . g . f .}=p_{I} \partial_{\sigma} \mathbf{x}^{I}, \quad \mathcal{H}_{T}^{\xi . g . f .}=\frac{1}{4 \pi \alpha^{\prime}}\left(\left(2 \pi \alpha^{\prime}\right)^{2} p_{I} g^{I J} p_{J}+\partial_{\sigma} \mathbf{x}^{I} g_{I J} \partial_{\sigma} \mathbf{x}^{J}\right)
$$

where $\mathbf{x}^{I}=\left(r, x^{i}, \chi, y^{a}\right), I=1, \ldots, 8$ and components of the metric $g_{I J}$ and two form fields $b_{t I}$ follow from (2.14), (2.18) and (2.19). Note that we again ignore terms $b_{I t} \partial_{\sigma} t$ that vanish in the gauge (3.14).

As usual we start with the calculation of Poisson brackets of the gauge fixing functions (3.14) with $\mathcal{H}_{T}, \mathcal{H}_{S}$

$$
\begin{aligned}
& \left\{G_{t}(\sigma), \mathcal{H}_{T}\left(\sigma^{\prime}\right)\right\}=2 \pi \alpha^{\prime} g^{t \xi} p_{\xi}(\sigma) \delta\left(\sigma-\sigma^{\prime}\right), \\
& \left\{G_{t}(\sigma), \mathcal{H}_{S}\left(\sigma^{\prime}\right)\right\}=\partial_{\sigma} t(\sigma) \delta\left(\sigma-\sigma^{\prime}\right) \approx 0 \\
& \left\{G_{\xi}(\sigma), \mathcal{H}_{T}\left(\sigma^{\prime}\right)\right\}=\left(\left(2 \pi \alpha^{\prime} p_{t}-b_{t I} \partial_{\sigma} \mathbf{x}^{I}\right) g^{t \xi}+2 \pi \alpha^{\prime} p_{\xi} g^{\xi \xi}\right)(\sigma) \delta\left(\sigma-\sigma^{\prime}\right), \\
& \left\{G_{\xi}(\sigma), \mathcal{H}_{S}\left(\sigma^{\prime}\right)\right\}=\partial_{\sigma} \xi(\sigma) \delta\left(\sigma-\sigma^{\prime}\right) \approx m \delta\left(\sigma-\sigma^{\prime}\right) .
\end{aligned}
$$

Then the requirement that (3.14) are preserved during the time evolutions of the system uniquely determine the functions $f_{T}, f_{S}$ and we find

$$
\begin{aligned}
& f_{T}=\frac{1-2 \pi \alpha^{\prime} g^{t \xi} p_{\xi}}{2 \pi \alpha^{\prime} g^{t \xi} p_{\xi}} \\
& f_{S}=-\frac{1}{2 \pi \alpha^{\prime} m g^{t \xi} p_{\xi}}\left(\left(2 \pi \alpha^{\prime} p_{t}-b_{t I} \partial_{\sigma} \mathbf{x}^{I}\right) g^{t \xi}+2 \pi \alpha^{\prime} p_{\xi} g^{\xi \xi}\right) .
\end{aligned}
$$

Next steps are as in the previous section. Solving $\mathcal{H}_{S}=0$ we express $p_{\xi}$ as functions of the reduced phase space variables $\mathbf{x}^{I}$

$$
p_{\xi}=-\frac{1}{m} \mathcal{H}_{S}^{\xi . g . f .}
$$


Solving the constraint $\mathcal{H}_{T}=0$ we find that the gauge fixed Hamiltonian $\mathcal{H}^{\xi . g . f .}=-p_{t}$ takes the form

$$
\begin{aligned}
\mathcal{H}^{\xi . g . f .} & =-\frac{1}{2 \pi \alpha^{\prime}} b_{t I} \partial_{\sigma} \mathbf{x}^{I}+\frac{1}{2} \frac{g^{\xi \xi}}{g^{t \xi}} p_{\xi}+\frac{1}{2 \pi \alpha^{\prime} g^{t \xi} p_{\xi}} \mathcal{H}_{T}^{\xi . g . f .}= \\
& =-\frac{1}{2 \pi \alpha^{\prime}} b_{t I} \partial_{\sigma} \mathbf{x}^{I}+\frac{1}{2 m} \frac{g_{t t}}{g_{t \xi}} \mathcal{H}_{S}^{\xi . g . f .}-\frac{m g_{t \xi}}{2 \pi \alpha^{\prime}} \frac{\mathcal{H}_{T}^{\xi . g . f .}}{\mathcal{H}_{S}^{\xi . g . f .}},
\end{aligned}
$$

where in the final step we used (3.20). Clearly the Hamiltonian (3.21) is well defined on condition that $\mathcal{H}_{S}^{\xi . g . f .} \neq 0$. In particular, this result implies that there do not exist pure time dependent solutions.

It is also instructive to determine the Lagrangian density from the gauge fixed form of the Hamiltonian density (3.21). The first step is to find time derivative of $\mathbf{x}^{I}$ using the canonical equation of motion

$$
\partial_{\tau} \mathbf{x}^{I}=\left\{\mathbf{x}^{I}, H^{\xi . g . f .}\right\}=\frac{1}{2 m} \frac{g_{t t}}{g_{t \xi}} \partial_{\sigma} \mathbf{x}^{I}-m g_{t \xi} \frac{g^{I J} p_{J}}{\mathcal{H}_{S}^{\xi . g . f .}}+\frac{m g_{t \xi}}{2 \pi \alpha^{\prime}} \frac{\mathcal{H}_{T}^{\xi . g . f .}}{\left(\mathcal{H}_{S}^{\xi . g . f .}\right)^{2}} \partial_{\sigma} \mathbf{x}^{I} .
$$

Then after some algebra we find the Lagrangian density in the form

$$
\begin{aligned}
\mathcal{L}_{\xi . g . f .} & =\partial_{\sigma} \mathbf{x}^{I} p_{I}-\mathcal{H}^{\xi . g . f .}=\frac{1}{2 \pi \alpha^{\prime}} b_{t I} \partial_{\sigma} \mathbf{x}^{I}- \\
& -\frac{1}{2 \pi \alpha^{\prime}} \sqrt{\left.-\left(g_{t t}+\left(\partial_{\tau} \mathbf{x}^{I} \partial_{\tau} \mathbf{x}_{I}\right)\right)\left(\partial_{\sigma} \mathbf{x}^{I} \partial_{\sigma} \mathbf{x}_{I}\right)\right)+\left(m g_{t \xi}+\partial_{\tau} \mathbf{x}^{I} \partial_{\sigma} \mathbf{x}_{I}\right)^{2}} .
\end{aligned}
$$

In other words we reproduced the Nambu-Goto action for bosonic string in the gauge $t=\tau, \quad \xi=m \sigma$. The form of the Lagrangian density (3.23) again implies that it is not possible to find pure time dependent solution of the equation of motion. It will be interesting to find configuration that depends on $\tau$ and $\sigma$ and that is a solution of the equation of motion that follow from (3.23). We hope to return to this problem in future.

\section{Uniform Gauge $t=\tau, p_{\xi}=$ const}

In this section we construct the Hamiltonian for the bosonic string in the background 2.14) where the momentum $P_{\xi}$ is uniformly distributed along the string. In fact, since the background (2.14) is invariant under the shift $\xi^{\prime}=\xi+\delta \xi$ with $\delta \xi=$ const the standard arguments imply an existence of the conserved charge

$$
P_{\xi}=\int_{-r}^{r} d \sigma p_{\xi}
$$

where, as in previous section, we consider the closed string with $\sigma \in[-r, r]$. Following the seminal work [54] we impose the uniform gauge by introducing two gauge fixing functions

$$
G_{1}: t-\tau=0, \quad G_{2}: p_{\xi}-\mathbf{p}=0,
$$


where $\mathbf{p}=$ const. Since

$$
\begin{aligned}
& \left\{G_{1}(\sigma), \mathcal{H}_{T}\left(\sigma^{\prime}\right)\right\}=2 \pi \alpha^{\prime} g^{t \xi} p_{\xi}(\sigma) \delta\left(\sigma-\sigma^{\prime}\right) \approx 2 \pi \alpha^{\prime} g^{t \xi} \mathbf{p} \delta\left(\sigma-\sigma^{\prime}\right), \\
& \left\{G_{1}(\sigma), \mathcal{H}_{S}\left(\sigma^{\prime}\right)\right\}=\partial_{\sigma} t(\sigma) \delta\left(\sigma-\sigma^{\prime}\right) \approx 0, \\
& \left\{G_{2}(\sigma), \mathcal{H}_{T}\left(\sigma^{\prime}\right)\right\}=-\frac{1}{\pi \alpha^{\prime}} g_{t \xi}\left(\sigma^{\prime}\right) \partial_{\sigma^{\prime}} t\left(\sigma^{\prime}\right) \partial_{\sigma^{\prime}} \delta\left(\sigma-\sigma^{\prime}\right) \approx 0, \\
& \left\{G_{2}(\sigma), \mathcal{H}_{S}\left(\sigma^{\prime}\right)\right\}=-p_{\xi}\left(\sigma^{\prime}\right) \partial_{\sigma^{\prime}} \delta\left(\sigma-\sigma^{\prime}\right) \approx-\mathbf{p} \partial_{\sigma^{\prime}} \delta\left(\sigma-\sigma^{\prime}\right)
\end{aligned}
$$

we find that (4.2) together with (2.12) form the second class constraints. Further the requirement of the preservation of the gauge fixing functions (4.2) during the time evolution of the system implies following set of equations

$$
\begin{aligned}
\frac{d G_{1}}{d \tau} & =-1+\left(\frac{N_{T}}{\sqrt{\omega}}+f_{T}\right) 2 \pi \alpha^{\prime} g^{t \xi} \mathbf{p} \approx 0 \\
\frac{d G_{2}}{d \tau} & =-\left(\frac{1}{\pi \alpha^{\prime}} g_{t \xi}\left(\frac{N_{T}}{\sqrt{\omega}}+f_{T}\right)(r)+\mathbf{p}\left(N_{S}+f_{S}\right)(r)\right) \delta(\sigma-r)+ \\
& +\left(\frac{1}{\pi \alpha^{\prime}} g_{t \xi}\left(\frac{N_{T}}{\sqrt{\omega}}+f_{T}\right)(-r)+\mathbf{p}\left(N_{S}+f_{S}\right)(-r)\right) \delta(\sigma+r)+ \\
& +\partial_{\sigma}\left[\frac{1}{\pi \alpha^{\prime}} g_{t \xi}\left(\frac{N_{T}}{\sqrt{\omega}}+f_{T}\right)+\left(N_{S}+f_{S}\right) \mathbf{p}\right] \approx 0,
\end{aligned}
$$

using the Poisson brackets given in (4.3). Fixing the metric components as in (3.5) we find that the equations (4.4) imply

$$
f_{T}=\frac{1}{2 \pi \alpha^{\prime} g^{t \xi} \mathbf{p}}-1, \quad f_{S}=-\frac{g_{t \xi}^{2}}{\mathbf{p}^{2}} .
$$

Now with the help of the second gauge fixed function (4.2) we easily calculate the total conserved momentum $P_{\xi}$

$$
P_{\xi}=\int_{-r}^{r} d \sigma p_{\xi}=2 r \mathbf{p}
$$

and hence we find the relation between $r, \mathbf{p}$ and $P_{\xi}$

$$
r=\frac{1}{2 \mathbf{p}} P_{\xi}
$$

that will be useful below.

Let us proceed to the construction of the gauge fixed Hamiltonian. Solving the constraint $\mathcal{H}_{S}=0$ we find

$$
\partial_{\sigma} \xi=-\frac{1}{\mathbf{p}} \mathcal{H}_{S}^{\xi . g . f .} .
$$

Since $\xi$ is a periodic variable string can wrap this direction. In other words we should identify the end points of the string as

$$
\triangle \sigma=\xi(r)-\xi(-r)=m L,
$$


where $m$ is the winding number. Then using (4.8) we can rewrite this condition in the equivalent form

$$
\begin{aligned}
& \xi(r)-\xi(-r)=\int_{-r}^{r} d \sigma \partial_{\sigma} \xi= \\
= & -\frac{1}{\mathbf{p}} \int_{-r}^{r} d \sigma \mathcal{H}_{S}^{\xi . g . f .}=m L .
\end{aligned}
$$

Clearly all classical solutions have to obey this condition. As the last step we determine the form of the gauge fixed Hamiltonian density $\mathcal{H}^{\text {p.g.f. }}=-p_{t}$. Using the constraint $\mathcal{H}_{T}=0$ together with (5.7) we find that it takes the form

$$
\mathcal{H}^{p . g . f .}=-\frac{1}{2 \pi \alpha^{\prime}} b_{t I} \partial_{\sigma} \mathbf{x}^{I}-\frac{\mathbf{p}}{2} \frac{g_{t t}}{g_{t \xi}}+\frac{g_{t \xi}}{2 \pi \alpha^{\prime} \mathbf{p}} \mathcal{H}^{\xi . g . f .} .
$$

We see that the Hamiltonian is positive definite for $\mathbf{p}>0$. Further, as follows from (4.7) we can set $\mathbf{p}$ to be equal to $\mathbf{p}=\frac{1}{2 \pi \alpha^{\prime}}$ by rescaling $r$.

Let us now find the Lagrangian density from the Hamiltonian density (4.11). Since

$$
\partial_{\tau} \mathbf{x}^{I}=\left\{\mathbf{x}^{I}, H^{p . g . f .}\right\}=2 \pi \alpha^{\prime} g_{t \xi} g^{I J} p_{J}
$$

and since $g^{I J}$ is invertible we can express $p_{I}$ as functions of $\partial_{\tau} \mathbf{x}, \mathbf{x}$ and consenquently the Lagrangian density takes the form

$$
\begin{aligned}
& \mathcal{L}^{p . g . f .}=\partial_{\tau} \mathbf{x}^{I} p_{I}-\mathcal{H}^{p . g . f .}= \\
= & \frac{1}{4 \pi \alpha^{\prime}}\left(\frac{1}{g_{t \xi}} g_{I J} \partial_{\tau} \mathbf{x}^{I} \partial_{\tau} \mathbf{x}^{J}-g_{t \xi} g_{I J} \partial_{\sigma} \mathbf{x}^{I} \partial_{\sigma} \mathbf{x}^{J}\right)+\frac{1}{2 \pi \alpha^{\prime}} b_{t I} \partial_{\sigma} \mathbf{x}^{I}+\frac{1}{2} \frac{g_{t t}}{g_{t \xi}} .
\end{aligned}
$$

It is instructive to study the the dynamics of the radial mode. Using the form of the metric components

$$
g_{t t}=-\frac{R^{2}}{r^{4}}, \quad g_{t \xi}=\frac{R^{2}}{r^{2}}, \quad g_{r r}=\frac{R^{2}}{r^{2}}
$$

we find that the truncanted Lagrangian for $r$ is equal to

$$
\mathcal{L}_{r}=\frac{1}{4 \pi \alpha^{\prime}}\left(\partial_{\tau} r\right)^{2}-\frac{1}{2} \frac{1}{r^{2}}
$$

so that the equation of motion for $r$ takes the form

$$
-\frac{1}{2 \pi \alpha^{\prime}} \partial_{\tau} \partial_{\tau} r+\frac{1}{r^{3}}=0 .
$$

If we multiply this equation with $\partial_{\tau} t$ we can rewrite it as

$$
\partial_{\tau}\left[\frac{1}{4 \pi \alpha^{\prime}}\left(\partial_{\tau} t\right)^{2}+\frac{1}{2 r^{2}}\right]=0
$$


and consequently

$$
\left(\partial_{\tau} r\right)^{2}=4 \pi \alpha^{\prime}\left(E-\frac{1}{2 r^{2}}\right)
$$

where $E>0$ is a conserved energy of the string. The expression on the right side is positive for

$$
r>\frac{1}{\sqrt{2 E}} .
$$

It is easy to determine the solution of the equation 4.18

$$
r=\frac{1}{\sqrt{2 E}} \sqrt{1+8 \pi \alpha^{2} E^{2} t^{2}},
$$

where the initial condition was choosen such that for $\tau=0$ the string reaches its turning point at $r=\frac{1}{\sqrt{2 E}}$. In fact, according to the equation 4.20 the string with energy $E$ is localized at $r=\infty$ in infinity past $\tau=-\infty$, then moves towards to the boundary of the space, reaches its turning point at $r=\frac{1}{\sqrt{2 E}}$ and then moves to $r=\infty$ for $\tau=\infty$. In other words the string with positive energy cannot reach the boundary of the space at $r=0$. Clearly this result could have an impact on the formulation of the holographic relations between non-relativistic quantum field theories on the boundary and the string modes in the bulk of the non-relativistic background.

\section{Strings in Non-Extremal Non-Relativistic D3-brane Background}

In this section we briefly discuss the Hamiltonian dynamics of the string in non-extremal non-relativistic D3-brane background [22, 26]

$$
\begin{aligned}
d s^{2}= & \left(\frac{R}{r}\right)^{2} \frac{1}{K}\left[-\left(\frac{g}{2}+\frac{2 f \triangle^{2}}{r^{2}}\right) d t^{2}-\frac{g}{2} d \xi^{2}+(1+f) d t d \xi+K\left(d x^{i}\right)^{2}\right]+ \\
+ & \left(\frac{R}{r}\right)^{2}\left[f^{-1} d r^{2}+r^{2}\left(\frac{1}{K}(d \chi+\mathcal{A})^{2}+d s_{P}^{2}\right)\right] \\
B= & \frac{\triangle R^{2}}{\sqrt{2} r^{2} K}(d \chi+\mathcal{A}) \wedge((1+f) d t+(1-f) d \xi) \\
& e^{\Phi}=\frac{1}{\sqrt{K}}
\end{aligned}
$$

where

$$
f=1+g, \quad K=1-\beta^{2} r^{2} g, \quad g=\frac{\rho_{H}^{4}}{r^{4}},
$$

where $\beta$ is a free parameter. It is expected that this background is dual to the nonrelativistic quantum field theory at finite temperature and with non-zero chemical potential [21, 22]. An important property of this metric (5.1) is that there is a non-zero component of 
metric $g_{\xi \xi}$ and also non-zero component of the NS two form field $b_{\chi \xi}, b_{a \xi}$. As a consequence of this fact we find that the components of metric $g^{t t}, g^{t \xi}, g^{\xi \xi}$ are equal to

$$
g^{t t}=\frac{g_{\xi \xi}}{g_{t t} g_{\xi \xi}-g_{t \xi} g_{t \xi}}, \quad g^{t \xi}=g^{\xi t}=-\frac{g_{t \xi}}{g_{t t} g_{\xi \xi}-g_{t \xi} g_{t \xi}}, \quad g^{\xi \xi}=\frac{g_{\xi \xi}}{g_{t t} g_{\xi \xi}-g_{t \xi} g_{t \xi}} .
$$

Then the Hamiltonian density is equal to

$$
\mathcal{H}=\left(\frac{N_{T}}{\sqrt{\omega}}+f_{T}\right) \mathcal{H}_{T}+\left(N_{S}+f_{S}\right) \mathcal{H}_{S}+u_{N} \pi_{N}+u_{S} \pi_{S}+u_{\omega} \pi_{\omega}
$$

where

$$
\begin{aligned}
\mathcal{H}_{S} & =p_{t} \partial_{\sigma} t+p_{\xi} \partial_{\sigma} \xi+\partial_{\sigma} \mathbf{x}^{I} p_{I} \\
\mathcal{H}_{T} & =\frac{1}{4 \pi \alpha^{\prime}}\left(\left(2 \pi \alpha^{\prime} p_{t}-b_{t I} \partial_{\sigma} \mathbf{x}^{I}\right) g^{t t}\left(2 \pi \alpha^{\prime} p_{t}-b_{t J} \partial_{\sigma} \mathbf{x}^{J}\right)+\right. \\
& +2\left(2 \pi \alpha^{\prime} p_{\xi}-b_{\xi I} \partial_{\sigma} \mathbf{x}^{I}\right) g^{\xi t}\left(2 \pi \alpha^{\prime} p_{t}-b_{t J} \partial_{\sigma} \mathbf{x}^{J}\right)+ \\
& +\left(2 \pi \alpha^{\prime} p_{\xi}-b_{\xi I} \partial_{\sigma} \mathbf{x}^{I}\right) g^{\xi \xi}\left(2 \pi \alpha^{\prime} p_{\xi}-b_{\xi J} \partial_{\sigma} \mathbf{x}^{J}\right)+ \\
& +\left(2 \pi \alpha^{\prime} p_{I}-b_{I \xi} \partial_{\sigma} \xi-b_{I t} \partial_{\sigma} t\right) g^{I J}\left(2 \pi \alpha^{\prime} p_{J}-b_{J \xi} \partial_{\sigma} \xi-b_{J t} \partial_{\sigma} t\right)+ \\
& +g_{t t} \partial_{\sigma} t \partial_{\sigma} t+2 g_{t \xi} \partial_{\sigma} t \partial_{\sigma} \xi+g_{\xi \xi} \partial_{\sigma} \xi \partial_{\sigma} \xi+g_{I J} \partial_{\sigma} \mathbf{x}^{I} \partial_{\sigma} \mathbf{x}^{J} .
\end{aligned}
$$

Since the static gauge can be easily formulate in the Lagrangian formalism of given theory we restrict ourselves to the case of the uniform gauge defined as

$$
G_{1}: t-\tau=0, \quad G_{2}: p_{\xi}-\mathbf{p}=0 .
$$

Then we can proceed exactly in the same way as in previous section. Solving the constraint $\mathcal{H}_{S}=0$ we find

$$
\partial_{\sigma} \xi=-\frac{1}{\mathbf{p}} \partial_{\sigma} \mathbf{x}^{I} p_{I}
$$

and solving the constraint $\mathcal{H}_{T}=0$ for $p_{t}$ we find the gauge fixed Hamiltonian density $\mathcal{H}^{n . b . f .}=-p_{t}$ in the form

$$
\mathcal{H}^{n . b . f .}=-\frac{1}{2 \pi \alpha^{\prime}} b_{t I} \partial_{\sigma} \mathbf{x}^{I}-\frac{1}{2 \pi \alpha^{\prime}} \frac{g_{t \xi}}{g_{\xi \xi}}\left(1-b_{\xi I} \partial_{\sigma} \mathbf{x}^{I}\right)-\frac{1}{2 \pi \alpha^{\prime} g^{t t}} \sqrt{\mathbf{K}}
$$

where

$$
\begin{aligned}
\mathbf{K} & =-\frac{1}{g_{t t} g_{\xi \xi}-g_{t \xi}^{2}}\left[\left(1-b_{\xi I} \partial_{\sigma} \mathbf{x}^{I}\right)^{2}+4 \pi^{2} \alpha^{\prime 2} g_{\xi \xi}\left(p_{I}+\left(\partial_{\sigma} \mathbf{x}^{K} p_{K}\right) b_{I \xi}\right) g^{I J}\left(p_{J}+\left(\partial_{\sigma} \mathbf{x}^{L} p_{L}\right) b_{J \xi}\right)+\right. \\
& \left.+g_{\xi \xi}\left(\partial_{\sigma} \mathbf{x}^{I} p_{I}\right)\left(\partial_{\sigma} \mathbf{x}^{J} p_{J}\right)+g_{\xi \xi} g_{I J} \partial_{\sigma} \mathbf{x}^{I} \partial_{\sigma} \mathbf{x}^{J}\right],
\end{aligned}
$$

where we set $\mathbf{p}=\frac{1}{2 \pi \alpha^{\prime}}$ and used the equation (5.7). Note that now the gauge fixed Hamiltonian takes the square-root form as in the relativistic case which is in contrast with the gauge fixed Hamiltonian for string in non-relativistic extremal background. 
Clearly the same gauge fixed Hamiltonian (5.8) can be used for the description of the string in uniform gauge that moves in the background that is dual to the non-relativistic quantum field theory at zero temperature but with non-zero particle density. This background was derived in [22] and it takes remarkably simple form

$$
\begin{aligned}
d s^{2} & =\frac{1}{r^{2} \kappa}\left(-\frac{d t^{2}}{r^{2}}+2 d t d \xi+\Omega^{2} r^{4} d \xi^{2}\right)+\frac{\left(d x^{i}\right)^{2}+d r^{2}}{r^{2}} \\
B & =\frac{1}{r^{2} \kappa}\left(d t+2 \Omega^{2} r^{4} d \xi\right) \wedge(d \chi+\mathcal{A}) \\
\phi & =-\frac{1}{2} \ln \kappa
\end{aligned}
$$

where for simplicity we ignored the sphere directions and where $\kappa=1+\Omega^{2} r^{2}$ and where $\Omega$ is parameter that is related to the particle number density in dual field theory. This background possesses many interesting properties and certainly deserves to be studied further. We hope to return to the study of the dynamics of string in given background in future.

Acknowledgement: This work was supported by the Czech Ministry of Education under Contract No. MSM 0021622409.

\section{References}

[1] J. M. Maldacena, "The large $N$ limit of superconformal field theories and supergravity," Adv. Theor. Math. Phys. 2, 231 (1998) [Int. J. Theor. Phys. 38, 1113 (1999)] [arXiv:hep-th/9711200].

[2] S. S. Gubser, I. R. Klebanov and A. M. Polyakov, "Gauge theory correlators from non-critical string theory," Phys. Lett. B 428, 105 (1998) [arXiv:hep-th/9802109].

[3] E. Witten, “Anti-de Sitter space and holography," Adv. Theor. Math. Phys. 2, 253 (1998) [arXiv:hep-th/9802150].

[4] S. A. Hartnoll, "Lectures on holographic methods for condensed matter physics," arXiv:0903.3246 [hep-th].

[5] J. McGreevy, "Holographic duality with a view toward many-body physics," arXiv:0909.0518 [hep-th].

[6] C. R. Hagen, "Scale and conformal transformations in galilean-covariant field theory," Phys. Rev. D 5, 377 (1972).

[7] U. Niederer, "The maximal kinematical invariance group of the free Schrodinger equation," Helv. Phys. Acta 45, 802 (1972).

[8] M. Henkel, "Schrodinger invariance in strongly anisotropic critical systems," J. Statist. Phys. 75, 1023 (1994) [arXiv:hep-th/9310081].

[9] C. Duval, G. W. Gibbons and P. Horvathy, "Celestial Mechanics, Conformal Structures, and Gravitational Waves," Phys. Rev. D 43 (1991) 3907 [arXiv:hep-th/0512188]. 
[10] C. Duval and P. A. Horvathy, "Non-relativistic conformal symmetries and Newton-Cartan structures," arXiv:0904.0531 [math-ph].

[11] T. Mehen, I. W. Stewart and M. B. Wise, "Conformal invariance for non-relativistic field theory," Phys. Lett. B 474, 145 (2000) [arXiv:hep-th/9910025].

[12] D. T. Son and M. Wingate, "General coordinate invariance and conformal invariance in nonrelativistic physics: Unitary Fermi gas," Annals Phys. 321, 197 (2006) [arXiv:cond-mat/0509786].

[13] C. Leiva and M. S. Plyushchay, "Conformal symmetry of relativistic and nonrelativistic systems and AdS/CFT correspondence," Annals Phys. 307 (2003) 372 [arXiv:hep-th/0301244].

[14] F. Correa, V. Jakubsky and M. S. Plyushchay, "Aharonov-Bohm effect on AdS $S_{2}$ and nonlinear supersymmetry of reflectionless Poschl-Teller system," Annals Phys. 324 (2009) 1078 [arXiv:0809.2854 [hep-th]].

[15] Y. Nishida and D. T. Son, "Nonrelativistic conformal field theories," Phys. Rev. D 76, 086004 (2007) [arXiv:0706.3746 [hep-th]].

[16] D. T. Son, "Toward an AdS/cold atoms correspondence: a geometric realization of the Schroedinger symmetry," Phys. Rev. D 78, 046003 (2008) [arXiv:0804.3972 [hep-th]].

[17] K. Balasubramanian and J. McGreevy, "Gravity duals for non-relativistic CFTs," Phys. Rev. Lett. 101, 061601 (2008) [arXiv:0804.4053 [hep-th]].

[18] W. D. Goldberger, "AdS/CFT duality for non-relativistic field theory," JHEP 0903, 069 (2009) [arXiv:0806.2867 [hep-th]].

[19] J. L. F. Barbon and C. A. Fuertes, "On the spectrum of nonrelativistic AdS/CFT," JHEP 0809, 030 (2008) [arXiv:0806.3244 [hep-th]].

[20] C. P. Herzog, M. Rangamani and S. F. Ross, "Heating up Galilean holography," JHEP 0811, 080 (2008) [arXiv:0807.1099 [hep-th]].

[21] J. Maldacena, D. Martelli and Y. Tachikawa, "Comments on string theory backgrounds with non-relativistic conformal symmetry," JHEP 0810, 072 (2008) [arXiv:0807.1100 [hep-th]].

[22] A. Adams, K. Balasubramanian and J. McGreevy, "Hot Spacetimes for Cold Atoms," JHEP 0811, 059 (2008) [arXiv:0807.1111 [hep-th]].

[23] P. Kovtun and D. Nickel, "Black holes and non-relativistic quantum systems," Phys. Rev. Lett. 102, 011602 (2009) [arXiv:0809.2020 [hep-th]].

[24] S. A. Hartnoll and K. Yoshida, "Families of IIB duals for nonrelativistic CFTs," JHEP 0812, 071 (2008) [arXiv:0810.0298 [hep-th]].

[25] M. Schvellinger, "Kerr-AdS black holes and non-relativistic conformal QM theories in diverse dimensions," JHEP 0812, 004 (2008) [arXiv:0810.3011 [hep-th]].

[26] L. Mazzucato, Y. Oz and S. Theisen, "Non-relativistic Branes," JHEP 0904, 073 (2009) [arXiv:0810.3673 [hep-th]].

[27] M. Rangamani, S. F. Ross, D. T. Son and E. G. Thompson, "Conformal non-relativistic hydrodynamics from gravity,” JHEP 0901, 075 (2009) [arXiv:0811.2049 [hep-th]].

[28] M. Alishahiha, R. Fareghbal, A. E. Mosaffa and S. Rouhani, "Asymptotic symmetry of geometries with Schrodinger isometry," arXiv:0902.3916 [hep-th]. 
[29] D. Martelli and Y. Tachikawa, "Comments on Galilean conformal field theories and their geometric realization," arXiv:0903.5184 [hep-th].

[30] S. F. Ross and O. Saremi, "Holographic stress tensor for non-relativistic theories," arXiv:0907.1846 [hep-th].

[31] S. Kachru, X. Liu and M. Mulligan, "Gravity Duals of Lifshitz-like Fixed Points," Phys. Rev. D 78, 106005 (2008) [arXiv:0808.1725 [hep-th]].

[32] M. Blau, J. Hartong and B. Rollier, "Geometry of Schroedinger Space-Times, Global Coordinates, and Harmonic Trapping," JHEP 0907, 027 (2009) [arXiv:0904.3304 [hep-th]].

[33] D. Yamada, "Thermodynamics of Black Holes in Schroedinger Space," Class. Quant. Grav. 26, 075006 (2009) [arXiv:0809.4928 [hep-th]].

[34] N. Bobev, A. Kundu and K. Pilch, "Supersymmetric IIB Solutions with Schródinger Symmetry," JHEP 0907 (2009) 107 [arXiv:0905.0673 [hep-th]].

[35] N. Bobev and A. Kundu, "Deformations of Holographic Duals to Non-Relativistic CFTs," JHEP 0907 (2009) 098 [arXiv:0904.2873 [hep-th]].

[36] A. Bagchi and R. Gopakumar, "Galilean Conformal Algebras and AdS/CFT," JHEP 0907, 037 (2009) [arXiv:0902.1385 [hep-th]].

[37] U. H. Danielsson and L. Thorlacius, "Black holes in asymptotically Lifshitz spacetime," JHEP 0903, 070 (2009) [arXiv:0812.5088 [hep-th]].

[38] G. Bertoldi, B. A. Burrington and A. Peet, "Black Holes in asymptotically Lifshitz spacetimes with arbitrary critical exponent," arXiv:0905.3183 [hep-th].

[39] G. Bertoldi, B. A. Burrington and A. W. Peet, "Thermodynamics of black branes in asymptotically Lifshitz spacetimes," arXiv:0907.4755 [hep-th].

[40] M. Sakaguchi and K. Yoshida, "More super Schrodinger algebras from psu(2,2-4)," JHEP 0808, 049 (2008) [arXiv:0806.3612 [hep-th]].

[41] W. Y. Wen, "AdS/NRCFT for the (super) Calogero model," arXiv:0807.0633 [hep-th].

[42] Y. Nakayama, "Index for Non-relativistic Superconformal Field Theories," JHEP 0810, 083 (2008) [arXiv:0807.3344 [hep-th]].

[43] D. Minic and M. Pleimling, "Non-relativistic AdS/CFT and Aging/Gravity Duality," Phys. Rev. E 78, 061108 (2008) [arXiv:0807.3665 [cond-mat.stat-mech]].

[44] C. Duval, M. Hassaine and P. A. Horvathy, "The geometry of Schródinger symmetry in gravity background/non-relativistic CFT," Annals Phys. 324, 1158 (2009) [arXiv:0809.3128 [hep-th]].

[45] A. Akhavan, M. Alishahiha, A. Davody and A. Vahedi, "Non-relativistic CFT and Semi-classical Strings," JHEP 0903 (2009) 053 [arXiv:0811.3067 [hep-th]].

[46] M. Sakaguchi, "Super Galilean conformal algebra in AdS/CFT," arXiv:0905.0188 [hep-th].

[47] A. Bagchi and I. Mandal, "Supersymmetric Extension of Galilean Conformal Algebras," arXiv:0905.0580 [hep-th].

[48] M. Alishahiha, A. Davody and A. Vahedi, "On AdS/CFT of Galilean Conformal Field Theories," JHEP 0908 (2009) 022 [arXiv:0903.3953 [hep-th]]. 
[49] A. Bagchi and I. Mandal, "On Representations and Correlation Functions of Galilean Conformal Algebras," arXiv:0903.4524 [hep-th].

[50] A. Volovich and C. Wen, "Correlation Functions in Non-Relativistic Holography," JHEP 0905 (2009) 087 [arXiv:0903.2455 [hep-th]].

[51] A. Akhavan, M. Alishahiha, A. Davody and A. Vahedi, "Fermions in non-relativistic AdS/CFT correspondence," arXiv:0902.0276 [hep-th].

[52] A. Donos and J. P. Gauntlett, "Supersymmetric solutions for non-relativistic holography," JHEP 0903 (2009) 138 [arXiv:0901.0818 [hep-th]].

[53] Y. Nakayama and S. J. Rey, "Observables and Correlators in Nonrelativistic ABJM Theory," JHEP 0908 (2009) 029 [arXiv:0905.2940 [hep-th]].

[54] G. Arutyunov and S. Frolov, "Integrable Hamiltonian for classical strings on $A d S_{5} x S^{5}$," JHEP 0502 (2005) 059 [arXiv:hep-th/0411089].

[55] G. Arutyunov and S. Frolov, "Foundations of the $A d S_{5} x S^{5}$ Superstring. Part I," J. Phys. A 42 (2009) 254003 [arXiv:0901.4937 [hep-th]].

[56] A. A. Tseytlin, "Spinning strings and AdS/CFT duality," arXiv:hep-th/0311139.

[57] A. A. Tseytlin, "Semiclassical strings and AdS/CFT," arXiv:hep-th/0409296.

[58] J. M. Maldacena, "Wilson loops in large N field theories," Phys. Rev. Lett. 80 (1998) 4859 [arXiv:hep-th/9803002].

[59] S. J. Rey, S. Theisen and J. T. Yee, "Wilson-Polyakov loop at finite temperature in large $N$ gauge theory and anti-de Sitter supergravity," Nucl. Phys. B 527, 171 (1998) [arXiv:hep-th/9803135].

[60] S. J. Rey and J. T. Yee, "Macroscopic strings as heavy quarks in large $N$ gauge theory and anti-de Sitter supergravity," Eur. Phys. J. C 22, 379 (2001) [arXiv:hep-th/9803001].

[61] J. Govaerts, "The quantum geometer's universe: Particles, interactions and topology," arXiv:hep-th/0207276.

[62] J. Govaerts, "Hamiltonian Quantization And Constrained Dynamics," SPIRES entry Leuven, Belgium: Univ. Pr. (1991) 371 p. (Leuven notes in mathematical and theoretical physics, B4)

[63] M. Henneaux and C. Teitelboim, "Quantization of gauge systems," SPIRES entry Princeton, USA: Univ. Pr. (1992) $520 p$ 